(Aus der III. medizinischen Abteilung des Prof. H. Schlesinger im allgemeinen Krankenhause in Wien.)

\title{
Zur Kenntnis der Bewegungsstörungen der Pseudosklerose.
}

\author{
Von \\ Josef Gerstmann und Paul Schilder, \\ Assistenten der psychiatrischen Klinik.
}

(Eingegangen am 7. März 1920.)

Trotz des großen Interesses, das für Erkrankungen des extrapyramidalen motorischen Systems, insbesondere seit den Publikationen von Wilson und Vogt, besteht, liegen genauer eklinische Analysen dieser Motilitätsstörungen noch nicht in genügender Anzahl vor. Dies gilt auch von den Motilitätsstörungen der Pseudosklerose, wobei wir mit diesem Namen auch die Gruppe der Wilsonschen Fälle mit umfassen. Der Tremor bzw. das Wackeln der Pseudosklerose ist zwar von Strü mpell erschöpfend beschrieben. Unsere Kenntnisse über die bei dieser Krankheitsgruppe vorkommenden Hypertonien ist jedoch noch lückenhaft. Wilson selbst und etwa gleichzeitig Oppenheim und C. Vogt haben die Abgrenzung dieser Hypertonien gegenüber den Hypertonien bei Pyramidenbahnerkrankungen in ausreichender Weise gegeben. Eine vollständige klinische Analyse dieser Motilitätsstörungen wird jedoch in ihren Arbeiten ebenso vermißt wie in den Ausführungen S tr ü m pells ${ }^{1}$ ) über den ,,amyostatischen Symptomenkomplex“. Im allgemeinen werden die extrapyramidalen Hypertonien zu wenig differenziert. So wird die Hypertonie der Pseudosklerose mit der der Paralysis agitans zusammen geworfen. Dieser Umstand macht eine eingehendere Analyse der Hypertonie bei den einschlägigen Fällen erforderlich. Bisher sind diesbezügliche Untersuchungen unseres Wissens nur in der Arbeit von Söderbergh ${ }^{2}$ ) und in der von Economo-Schilder ${ }^{3}$ ) zufinden. Wir selbst haben vor kurzem einen der Pseudosklerose ähnlichen Krankheitstypus ebenfalls in dieser Hinsicht näher analysiert ${ }^{4}$ ).

Ein auf der III. medizinischen Abteilung beobachteter und von uns'wiederholt untersuchter Fall von Wilsonscher Krankheit gibt

1) Dtsch. Zeitschr. f. Nervenheilk. 54. 1916.

2) Arkiv för inre Medizin 51, H. 1, Nr. 7.

3) Diese Zeitschr. 55. 1920.

4) Diese Zeitschr. 54. 1920.

z. t. d. g. Neur. u. Psych. 0 . LVIII. 
uns neuerlich Veranlassung zur Vornahme einer eingehenden Analyse dieser Motilitätsstörungen. Die uns von der Abteilung des Professors Schlesinger, der diesen Fall vor einigen Wochen in der ,Gesellschaft für innere Medizin" demonstrierte, freundlichst zur Verfügung gestellten Krankheitsgeschichte lautet kurz folgendermaßen:

Stefan Kolber, 22 Jahre alt, Eisendreher, aufgenommen auf die III. med. Abt. anfangs Dezember 1919.

Eltern leben und sind gesund; ebenso ein Bruder; keine sonstigen Geschwister; keine Konsanguinität, keine Nervenkrankheiten in der Familie.

Von Kinderkrankheiten Schafblattern und Masern. Pat. hat immer gut gelernt, war ausgelernter Eisendreher, hat viel Sport betrieben. Immer gesund gewesen.

Die jetzige Erkrankung begann vor ungefähr 5 Jahren, angeblich im Anschluß an eine Ohrfeige, die er erhalten hatte; er sei 2 Tage ohnmächtig gewesen. Ohnmachtszustände hätten sich noch nachher durch kurze Zeit wiederholt. Habe damals auch eine, vorübergehende Hörstörung gehabt, außerdem Schwindelgefühl. Ein halbes Jahr später bemerkte die Mutter des Pat., daß er beim Gehen die Füße am Boden schleifte, leicht niederfiel und dann nur schwer und mühsam aufstehen konnte. Er wurde auch bẹim Arbeiten ungeschickt, hatte Zittern in den Händen und wurde schließlich vor 4 Jahren ganz arbeitsunfähig. Das Zittern machte sich besonders beim Schreiben geltend. Krampfanfälle wurden nie beobachtet. Seit 3 Jahren kann er nicht mehr gehen, kann mit den Händen nicht mehr zweckmäßig hantieren, kann nicht mehr allein essen. Sprache zunehmend schlechter geworden, seit ca. 1 Jahr Unfähigkeit zu sprechen.

Die Intelligenz hat nicht gelitten., Kein Potus. Kein Nicotin. Lues negiert.

11. XII. Status praesens: Pat. nimmt fast ständig. Rückenlage ein und bietet einen eigentümlichen Gesichtsausdruck dar: Die Augen weit aufgerissen, der Mund sehr weit und ständig geöffnet, das Gesicht von starrem Ausdruck, zeitweilig von einem krampfhaften Lachen verzogen. Pat. kann gar nicht sprechen, stößt zeitweilig einen unartikulierten Laut aus, verhält sich aber meist ruhig. Die lebhaft hin und her wandernden Augen verraten seine Anteilnahme an der Umgebung. Pat. wendet auch häufig den Kopf, oder hebt denselben, um besser zu sehen.

Der Kopf zeigt beinahe ständig ein Zittern. Auch die Extremitäten, namentlich die Hände, weisen ein solches auf. Von Zeit zu Zeit werden schüttelnde Bewegungen der Hände, oder grobe Fingerbewegungen, an choreatische entfernt erinnernd, vorgenommen, dann folgt eine minutenlange Pause, bis neuerlich Bewegungen einsetzen. Bei dem Versuche, die Lage der Extremitäten zu ändern, aktiv oder passiv, nehmen die Schüttelbewegungen stark zu und verbreitern sich über den ganzen Körper. Es besteht eine schwere, Agonisten und Antagonisten betreffende, tonische Starre der Extremitäten, welche passiv nur sehr schwer überwunden werden kann und an den oberen wie unteren Extremitäten ausgebildet ist. Auch die Rumpfmuskulatur ist sehr gespannt. Die Möglichkeit von aktiven Bewegungen ist vorhanden. Pat. greift nach Gegenständen und hält sie mit großer Kraft fest, dabei Intentionstremor. Muskelatrophien sind weder an den oberen noch an den unteren Extremitäten nachweisbar.

Die gewöhnliche krampfhafte Haltung ist an den oberen Extremitäten die Beugestellung; zur Zeit der Untersuchung wird die linke untere Extremität dauernd gestreckt, die rechte in Beugestellung gehalten.

Die Patellarreflexe auslösbar und lebhaft, Fußklonus auf beiden Seiten nachweisbar. 
Babinskisches Zehen- und $O$ ppenheimsches Unterschenkelphänomen gehen plantar; Fluchtreflex nicht ausgebildet; die Bauchdeckenreflexe sind vorhanden, Skrotalreflexe lebhaft.

Die Sehnenreflexe an den oberen Extremitäten scheinen nicht gesteigert zu sein.

Die Prüfung der elektrischen Erregbarkeit ergibt im wesentlichen keine Abnormitäten, speziell keine Zeichen myotonischer Reaktion. Auch mechanische Muskelerregbarkeit normal.

Es besteht ausgesprochene vasomotorische Übererregbarkeit und zwar Dermographia rubra et alba. An den unteren Extremitäten ist außerdem trichopilarer Tetanus vorhanden, während er am Rumpfe und an den oberen Extremitäten fehlt.

Nach Anlegung einer Stauungsbinde treten zahlreiche kleinste Ekchymosen auf.

Pat. läßt Harn unter sich, ist seit den 3 Tagen seines Spitalsaufenthaltes obstipiert. (Späterhin verlor sich die Inkontinenz.)

Auf Berührungen und Stiche reagiert der Pat., wo immer man dieselben appliziert; eine genauere Sensibilitätsprüfung ist nicht durchführbar.

Brachycephaler Schädel, sonst ohne Besonderheiten, nicht klopf- und druckempfindlich; die Augenbewegungen sind frei; die Pupillen gleich weit, reagieren auf Licht und Akkommodation; der Augenhintergrund normal; kein Nystagmus; kein grünlicher Ring um die Cornea.

Stiche werden stets überall im Gesicht unangenehm empfunden. Cornealreflexe lebhaft.

In der Gegend der Masseteren fühlt man eine brettharte Anspannung der Muskulatur; jedoch kann es sich nicht um Anspannung der Masseteren handeln, da der Mund konstant maximal weit offen gehalten wird; dabei rutscht der Unterkiefer nach vorne; drückt man den Unterkiefer nach oben, so schnappen die Gelenkköpfe mit hörbarem Gerh̆usch in die Pfanne ein. Unterkieferreflex nicht auslösbar.

Die beiden Faciales sind gleich gut innerviert, und zwar sowohl Mund- als Augen- und Stirnfacialis.

Ohrenbef und (Dozent Beck): Trommelfell und Mittelohr beiderseits normal, am rechten Trommelfell keine Zeichen eines bestehenden oder überstandenen Traumas. Gehör, mit Lärmapparat geprüft, beiderseits normal (der Kranke kommt den an ihn gerichteten Aufforderungen nach). Gehör für hohe und tiefe Stimmgabeln normal. Kein spontaner Nystagmus. Zeigeversuch und Vestibularprüfung nicht durchführbar. Kehlkopf normal innerviert. - Erst nach zwei Irrigatoren kalten Wassers Nystagmus, keine Schwindelerscheinungen, kein Erbrechen, keine Übelkeiten. Vom linken Ohr dauert der Nystagmus ca. 4 Minuten, auch vom rechten Ohr ist kalorischer Nystagmus erst nach langer Spülung mit kaltem Wasser auslösbar, dauert $3 \frac{1}{2}$ Minuten. Kein Schwindelgefühl, kein Erbrechen.

Die Zunge liegt zumeist unbeweglich am Boden der Mundhöhle, jedoch kann der Kranke aktiv die Zunge bewegen, sogar ein wenig vorstrecken. Keine Atrophie der Zunge. Das Gaumensegel kann aktiv etwas gehoben werden. Gaumen- und Rachenreflexe sind sehr lebhaft. Trotzdem ist der ganze Rachen voll mit Speiseresten.

Pat muß sehr langsam gefüttert werden, weil er sich sonst verschluckt; Flüssigkeiten geraten ihm sogar zumeist in die Kehle. Regurgitation durch die Nase wurde bisher nicht beobachtet.

Läppisch-kindisches, dementes Verhalten. Häufiges Zwangslachen. Häufig Harninkontinenz.

Leber perkutorisch nicht vergrößert, fühlt sich bei der Palpation nicht derb an. Leberfunktionsprüfung: Dextroseversuch positiv (nur angedeutet), Galaktosever- 
such exquisit positiv (starke alimentäre Galaktosurie). Urobilinogen im Harn stark vermehrt. Urobilin nicht vorhanden. An den inneren Organen sonst keine Besonderheiten. Wassermannsche Reaktion im Blut negativ.

Zusammenfassung: Es handelt sich demnach um eine Erkrankung von schleichender Entwicklung, die bei einem aus gesunder Familie stammenden 22 jährigen Manne im 17. Lebensjahr einsetzte und zu einem Symptomenbilde geführt hat, welches im wesentlichen von zwei motorischen Erscheinungen beherrscht wird: Von einem den ganzen Körper betreffenden Rigor und von einem groben Tremor bzw. Wackeln bei Intentionen. Der Rigor kennzeichnet sich als extrapyramidaler dadurch, daß er Agonisten und Antagonisten gleichmäßig betrifft und die der Pyramidenbahnläsion zukommenden wesentlichen Reflexstörungen fehlen. (Kein Babinskisches Phänomen. Kein Oppenheim. Fußklonus allerdings vorhanden.) Lähmungen im engeren Sinn sind vicht vorhanden. Ein schweres bulbär-paralytisches Bild wird durch die Spannungszustände vorgetäuscht. Die Leberfunktionsprüfung ergibt Zeichen einer Leberschädigung (positiver Dextrose- und stark positiver Galaktoseversuch). Cornealring fehlt.

Die Koinzidenz des eigenartigen neurologischen Zustandsbildes mit Zeichen der Lebererkrankung läßt die Diagnose einer Krankheit aus der Pseudosklerosegruppe als gesichert erscheinen. Aus dem klinischen Bilde geht nicht hervor, ob wir es mit einer Pseudosklerose in engerem Sinne (d. $h$. diffuse Veränderungen im Gehirn und in den basalen Hirnganglien, jedoch ohne Einschmelzungsvorgänge im Striatum) oder mit einer Erkrankung nach dem Typus Homen, Anton, Wilson (d. h. mit Einschmelzungsvorgängen im Striatum und relativ wenig ausgesprochenen oder fehlenden Veränderungen im übrigen Gehirn) zu tun haben. Doch ist die letztere Annahme wahrscheinlicher, da nach den bisherigen Obduktionsbefunden bei der tonischen Starre häufiger Einschmelzungsvorgänge im Striatum gefunden wurden $\left.{ }^{1}\right)$. Diese an und für sich unsichere Differentialdiagnose ist jedoch für unsere späteren Ausführungen belanglos.

Die Analyse der Spannungen bei unserem Kranken ergab: Pat. hat Zeiten, in denen er mit entspannter Körpermuskulatur daliegt. Im Gesicht bleibt stets eine Starre, die sich in den weit aufgerissenen Augen und stets weitgeöffnetem Mund kundgibt. An den unteren Extremitäten geht die Entspannung zeitweise bis zur völligen Schlaffheit, besonders im rechten Bein, das manchmal geradezu wie das Heilbronnersche

1) Anmerkung zur Korrektur: Der Kranke ist inzwischen interkurrent an einer Grippe mit Pneumonie erkrankt und bald darauf gestorben. Bei der Obduktion wurden tatsächlich Einschmelzungsprozesse im Striatum, speziell im Linsenkern gefunden. Die histologische Bearbeitung des Falles erfolgt im hiesigen neurologischen Institut. 
„,breite Bein“ auseinanderfließt. An den oberen Extremitäten geht der Ruhetonus niemals unter die obere Grenze der Norm. Es gibt Zeiten, wo an den unteren Extremitäten auch ausgiebige passive Bewegungen an der Hypotonie nichts ändern. An den oberen Extremitäten hingegen rufen passive Bewegungen immer einen Hypertonus hervor, der in folgendem näher beschrieben werden soll.

Meist trifft man den Pat. mit gespannter Muskulatur an. Diese Spannung betrifft - wie erwähnt - Agonisten und Antagonisten gleichmäßig. Sie ist an den oberen Extremitäten ausgesprochener als an den unteren und links akzentuierter als rechts.

Der Hypertonus kann sehr beträchtliche Grade erreichen. Er läßt nur allmählich nach, wenn der Pat. sich in vollständiger Ruhe befindet. Passive Bewegungen stoßen zunächst auf Widerstand, der sich rasch löst, an den Beinen bis zur oben beschriebenen Hypotonie. Der Hypertonus kann auf verschiedene Art und Weise geweckt werden: 1. durch aktive Bewegungen, 2. durch brüske passive, manchmal durch langsame passive Bewegungen, 3. durch Hautreize wie z. B. Prüfung des Bauchdeckenreflexes, 4. durch psychische Erregung. Es ist charakteristisch und wichtig hervorzuheben, daß der Hypertonus jäh und plötzlich einsetzt. Er fixiert dann die eben aktiv gewonnene Haltung. Passiv gegebene Stellungen werden nicht beibehalten. Tritt der Hypertonus ein, während eine Stellung passiv gegeben wird, so erzwingt er die in seinem Sinne gelegene Innervation, z. B. am linken Bein die Kniestreckung, auch wenn passiv eine Beugestellung gegeben worden war. Auch der Arm wird durch denselben meistens in Streckstellung fixiert. Häufiger überfällt der Hypertonus die aktiv bewegte Gliedmaße und fixiert sie. Wenn Pat. nach Gegenständen faßt, so gelingt es ihm sehr häufig nicht, die Hand wieder zu öffnen.

Die aktive Bewegung selbst setzt dann rasch ein, wenn sie bei entspannter Muskulatur erfolgt. Ist der Muskel gespannt, so bedarf Pat. einige Zeit zu der für die Bewegung nötigen Entspannung. Eine weitere Entspannung wird anscheinend durch die aktive Bewegung selbst erzielt. Manchmal fällt es dem Pat. schwer, eine Bewegung zu machen. Man hat den Eindruck, daß ihm Abwehr- und halb unwillkürliche Greifbewegungen besser gelingen als Bewegungen, die ihm aufgetragen werden. Die Bewegung selbst erfolgt sehr rasch und prompt, bis sie durch den plötzlich eintretenden Hypertonus gebremst wird.

Die aktive Bewegung ist mit einem Wackeln verbunden, das dadurch charakterisiert wird, daß es in einem groben Hin- und Herpendeln in der Richtung der geforderten Intention besteht. Es tritt bisweilen am Beginn, meist jedoch im Verlauf der Bewegung ein und verstärkt sich gegen das Ende derselben zu. Die Tremorerscheinungen sind an den oberen Extremitäten stärker ausgesprochen als an den unteren, wo sie bisweilen 
gänzlich zurücktreten. Der Tremor setzt an den Armen vorwiegend in den Handgelenken an, an den Beinen im Bereich der Oberschenkelmuskulatur.

Tremor und Hypertonus zeigen als charakteristisches Merkmal die Tendenz zur Irradiation. Wird z. B. durch brüske passive Bewegung am linken Bein ein Streckhypertonus hervorgerufen, so spannt sich die gesamte Becken- und Bauchmuskulatur und auch die Muskulatur des anderen Beines an. Die Ausbreitung ist dabei eine ziemlich regellose. So rufen z. B. Armbewegungen, Mitspannungen bald im rechten bald im linken Bein hervor. Am leichtesten spricht im allgemeinen die Oberschenkelmuskulatur an, die bei Hautreizen und bei Prüfung der Hautreflexe fast allein in Mitspannung gerät. Da die Mitspannungen persistieren können, kann unter Umständen durch einen Reiz der ganze Körper in einen starren Spannungszustand geraten. Besonders auffallend ist, daß die Mundöffner sehr leicht in extreme Mitspannung geraten.

Die Wackelbewegungen irradiieren nach denselben Gesetzmäßigkeiten wie die Starre, so daß durch eine Bewegung der ganze Körper in Zittern geraten kann. Dieses kann dann bestehen bleiben, wodurch es ebenso zu Dauertremoren wie zu Dauerspannungen kommt.

Der Versuch des Aufrichtens und Gehens löst besonders leicht Spannungen und Zittererscheinungen am Gesamtkörper aus.

Es besteht eine ausgesprochene Verarmung an spontanen Bewegungsantrieben, was besonders im Gesicht auffällig ist. Doch können auch die Beine sehr lange in unbequemer Stellung liegen, ohne daß der Pat. den Versuch macht, die Stellung abzuändern. Eine Prüfung der groben Kraft ist beim Pat. nicht möglich. Man gewinnt aber den Eindruck, daß der Pat. jede Bewegung mit guter Kraft durchführen kann.

Das Ausfahren bei plötzlichem Nachlassen des Widerstandes und der Rückstoß sind eher lebhafter als in der Norm.

Es erscheint uns zunächst als sehr wesentlich, daß der Hypertonus unseres Falles reflektorisch auslösbar ist. Es entspricht dies dem Befund im Falle von Söderbergh und in dem von Economo-Schilder. Es dürfte ihm demnach eine allgemeine Bedeutung zukommen. Die Ergebnisse bei unserem Falle zeigen, wie aus diesem reflektorischen Tonus ein Dauertonus werden kann. Verzögert sich nämlich die Entspannung und tritt andererseits das Einschießen des Tonus bei relativ geringen Reizen ein, so wird es zu einer allgemeinen Dauerspannung kommen, da ja die Tendenz zur Irradiation für diese Art des Tonus charakteristisch ist. Sie wurde in allen drei genannten Fällen vorgefunden. In dem Falle von Söderbergh ist die reflektorische Entstehung des Hypertonus schon dadurch wahrscheinlich, da $B$ er bei jeder Aktion des Muskels eintritt. Söderbergh zieht übrigens mit Recht die Mitspannung 
bei Hautreizen als Beweis für die reflektorische Entstehung des Hypertonus mit heran.

Damit ist ein grundlegender Unterschied gegenüber dem Hypertonus der Paralysis agitans gegeben. Allerdings kennen wir nicht die anatomischen Differenzen, welche diesen verschiedenen klinischen Bildern entsprechen.

Eine eigenartige Charakteristik erhält der Hypertonus unseres Falles dadurch, daß er durch passive Bewegungen rasch zum Verschwinden gebracht werden kann. Wenn auch bei der Paralysis agitans passive Bewegungen entspannend wirken, so pflegt doch die Entspannung auch bei beginnenden Fällen nicht eine derart weitgehende zu sein, wie in unserem Falle und in dem Söderberghschen. In den Fällen von Wilson und in dem Falle von Eco no mo - Schilder war allerdings die so hervorgerufene Entspannung relativ gering, so daß dieser Unterschied offenbar kein durchgreifender ist.

Ein besonderes Gewicht ist auch auf die Raschheit der Bewegung zu legen; eine Verlangsamung der Bewegungen, welche nicht durch Spannungen bedingt gewesen wäre, wurde bei unserem Falle und dem letztzitierten nicht beobachtet. Söderbergh spricht von langsamen Bewegungen, scheidet aber nicht primäre Langsamkeit von der durch Spannung bedingten.

In den drei verglichenen Fällen werden passiv gegebene Stellungen nicht beibehalten und nicht durch Spannungen fixiert. Bei der Paralysis agitans entfällt ja durch eine derartige Fixierung sehr häufig das plötzliche Ausfahren nach dem Nachlaß eines Widerstandes. Auch der Rückstoß bei der Paralysis agitans ist sehr gering oder fehlend (dabei dürfte allerdings die Langsamkeit der Bewegungen mitspielen). In unserem Falle und auch in dem Falle von Söderbergh war er etwa der Norm entsprechend, in dem von Economo-Schilder war er lebhaft gesteigert.

Auch in unserem Fall ist demnach ebenso wie in dem letztgenannten grundsätzlich eine andere Tonusstörung anzunehmen als bei der Paralysis agitans. Die Tonusstörung im Falle Söderberghs nähert sich der der beiden genannten Fälle.

Das rasche Nachlassen des Hypertonus nach passiven Bewegungen erinnert in auffallender Weise an die eigenartigen Spannungen der Ungeschickten und gewisser Neurotiker. Bei der Rückbildung eines postparoxysmalen Rigors einer Epileptischen hat der eine von uns (Schilder) diese Ähnlichkeit bereits beobachtet ${ }^{1}$ ). Man muß sich ja vorstellen, daß auch die funktionellen Spannungen darauf beruhen, daß somatische Apparate in Gang gesetzt werden. Es ist anzunehmen, daß auch der extrapyramidale motorische Apparat in diesen Fällen in anderer Weise

1) Wien. klin. Wochenschr. 1919, Nr. 32. 
zur Arbeit kommt, als in der Norm. Daß auf psychischem Wege somatische Mechanismen direkt beeinträchtigt werden können, entspricht Vorstellungen, die der andere von uns (Gerstmann) an anderer Stelle entwickelt hat ${ }^{1}$ ).

Das Zittern der Pseudosklerose muß schon deshalb als selbständige Störung gelten, weil es auch anatomisch gut untersuchte Fälle gibt, in denen das Zittern vollständig fehlt und die Starre das Bild beherrscht [z. B. der Fall von Econo $\mathrm{mo}^{2}$ )]. Die Hauptcharakteristica des Tremors unseres Falles sind: Das Schwingen in der Ebene der Intention und das Ubergreifen auf andere Muskelgebiete. Der Rhythmus ist dabei in sämtlichen Gebieten dann ungefähr gleich. Das Wackeln überdauert häufig die Intention, wodurch der Anschein eines Spontantremors zustande kommt. Der Tremor ist manchmal zu Beginn der Bewegung schon vorhanden und verstärkt sich gegen das Ende der Bewegung zu. Da die Ausbreitung des Tremors ebenso wie das Überdauern desselben über die Zeit der ursprünglichen Innervation an den Hypertonus erinnern, wäre es verlockend, den Tremor als einen dissoziierten Hypertonus anzusehen, wie dies ja auch Strü m pell getan hat. Doch ist unseres Erachtens zu erwägen, ob nicht doch engere Beziehungen zum Intentionstremor der multiplen Sklerose bestehen. Für diesen ist es wahrscheinlich, daß er auf einer Störung desjenigen Apparates beruht, welcher den Bewegungsabschluß sichert. Dieser an das Kleinhirn gebundene Apparat hat den Tonusapparaten gegenüber eine gewisse Selbständigkeit. Es wäre denkbar, daß der Tremor der Pseudosklerosefälle mit Erschwerungen der Inszenesetzung des Bewegungsentwurfes und insbesondere des Bewegungsabschlusses in Zusammenhang steht. Dafür spricht vornehmlich die Verstärkung des Tremors am Ende der Bewegung. Allerdings wäre es möglich, daß diese Verstärkung nur mit einem direkten Tonusmuskelfaktor in Zusammenhang steht. Wir konnten in unserem Falle nicht feststellen, ob die Größe des durchmessenen Weges auf die Verstärkung des Tremors am Ende der Bewegung von Einfluß ist. Nur wenn diese Verstärkung von der Größe des durchmessenen Weges unabhängig ist, würden wir von einer Störung des Bewegungsabschlusses sprechen (vgl. hierzu Arbeit von Gerstmann und Schilder). Wir möchten auf diese Fragestellung jedenfalls eindringlich aufmerksam machen.

Die Verstärkung des Tremors bei der Intention ist, von allen übrigen bekannten Unterschieden gegenüber dem Paralysis-agitans-Tremor abgesehen, charakteristisch. Denn bei der letzten Erkrankung hemmt die Willkürsinnervation das Zittern. Das gilt auch von den Zuckungen

1) Wien. med. Wochenschr. 1919, Nr. 19.

2) Diese Zeitschr. 48.1918. 
der Chorea minor. Gleichwohl springt bei diesen beiden Erkrankungen der Tremor und die Zuckung auf das Gesamtkörperbereich über.

Man könnte auch für die letztgenannten Erkrankungen annehmen, daß Tremor und Zuckung von Intentionen anderer Muskelgebiete her gespeist werden. Es würden sich dann Utbergänge zwischen Spontantremor und Spontanzuckung und Zuckungen, die sich nur bei Intentionen finden, leicht erklären lassen.

Nach den Beschreibungen Str ü mpells u. a. tritt in einer Reihe von Fällen das Wackeln auch bei statischen Leistungen stark hervor. Auch hat Strümpell das regellose Schwingen des Tremors in verschiedenen Ebenen hervorgehoben. Es scheinen auch hier Unterschiede zwischen den einzelnen Fällen zu existieren, denen aber wohl eine allgemeinere Bedeutung nicht zukommt.

Es hat sich also für den Rigor unseres Falles eine scharfe Charakteristik geben lassen, die ihn unterscheidet von dem Rigor der Paralysis agitans. Man darf diese beiden Typen extrapyramidaler Spannungen nicht miteinander vermengen. Es gibt natürlich auch Fälle, welche der Paralysis agitans klinisch nicht ohne weiteres zuzurechen sind und die doch den Rigortypus dieser Erkrankung mehr oder minder ausgesprochen zeigen. Hierher gehören z. B. die Geschwister H. der Publikation Str ü mpells, unser unlängst publizierter Fall, und die Fälle Försters (arteriosklerotische Starre $)^{1}$ ). Strümpell hatte ausschließlich diesen Typus vor Augen. Wir glauben, gezeigt zu haben, daß es noch einen zweiten Typus gibt.

1) Allg. Zeitschr. f. Psych. 66. 